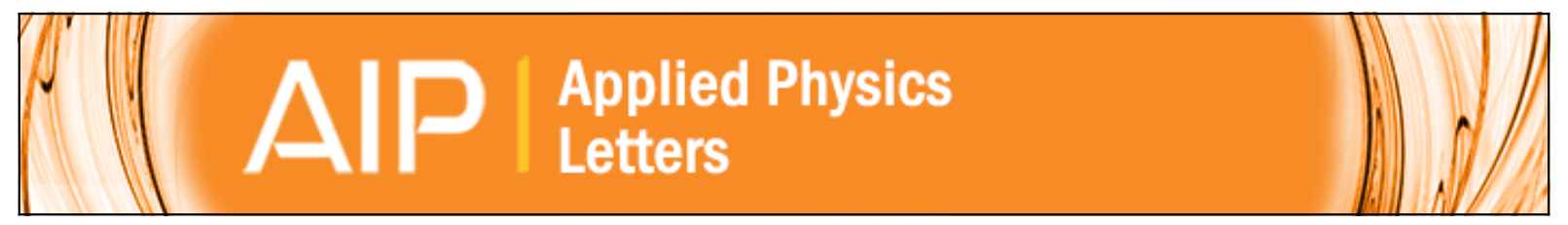

Polarization dynamics in vertical-cavity surface-emitting lasers with optical feedback through a quarter-wave plate

C. Masoller and N. B. Abraham

Citation: Applied Physics Letters 74, 1078 (1999); doi: 10.1063/1.123487

View online: http://dx.doi.org/10.1063/1.123487

View Table of Contents: http://scitation.aip.org/content/aip/journal/apl/74/8?ver=pdfcov

Published by the AIP Publishing 


\title{
Polarization dynamics in vertical-cavity surface-emitting lasers with optical feedback through a quarter-wave plate
}

\author{
C. Masoller \\ Instituto de Física, Facultad de Ciencias, Universidad de la República, Igua 4225, Montevideo 11400, \\ Uruguay and Department of Physics, Bryn Mawr College, Bryn Mawr, Pennsylvania 19010-2899
}

\author{
N. B. Abraham \\ Department of Physics, Bryn Mawr College, Bryn Mawr, Pennsylvania 19010-2899 and DePauw \\ University, Greencastle, Indiana 46135-0037
}

(Received 13 July 1998; accepted for publication 8 December 1998)

\begin{abstract}
Square-wave switching of the intensities of the orthogonal linearly polarized components of the output of a vertical-cavity surface-emitting laser (VCSEL) found previously in experiments with polarization-changing optical feedback, is not found in rate equation models incorporating only birefringence and gain anisotropy, but is found in the model for VCSELs developed by San Miguel, Feng, and Moloney [M. San Miguel, Q. Feng, and J. V. Moloney, Phys. Rev. A 52, 1729 (1995)]. The dynamics is sensitive to both the feedback strength and the relaxation rate of the magnetization in the quantum well sublevels. (C) 1999 American Institute of Physics. [S0003-6951(99)03806-1]
\end{abstract}

The output from a cylindrical vertical-cavity surfaceemitting laser (VCSEL) is usually linearly polarized along one of the two orthogonal directions given by the crystal axes or along stress anisotropies. For many experiments, when the excitation is just above the lasing threshold, one linear polarization dominates; and when the pump current is increased to about $20 \%-30 \%$ above threshold, the emission switches to the orthogonal linear polarization. ${ }^{1}$ The two linearly polarized states typically have different frequencies split by a few GHz by the material birefringence. The change of polarization state has attracted considerable interest because of its potential applications in optical switching. The polarization-state selection, the polarization dynamics, and the polarization switching in VCSELs can be changed by optical injection, ${ }^{2}$ current modulation, ${ }^{3}$ and by polarizationsensitive optical feedback. ${ }^{1,4}$

Here, we consider the behavior of a VCSEL under polarization-changing optical feedback, simulating the experimental situation described in Ref. 1. The external cavity is formed by the VCSEL and a partial reflector, and a quarter-wave plate is inserted inside the cavity with its axes rotated by $45^{\circ}$ with respect to the polarization basis states of the VCSEL. This type of feedback reinjects orthogonal linearly polarized emission into the laser if the initial emission is linearly polarized.

Key experimental results were sinusoidal modulation and square-wave switching of the intensities of the orthogonal linearly polarized components of the laser output. While the sinusoidal modulation is explained by simple models including birefringence and gain anisotropy, these models fail to explain the square-wave switching phenomenon. For this reason, we base our study on the model for VCSELs developed by San Miguel, Feng, and Moloney, ${ }^{5,6}$ which incorporates the cavity and material properties and which takes into account the phase dynamics of the electric field.

The rate equations of San Miguel, Feng, and Moloney for a VCSEL operating in its fundamental transverse mode, modified to include feedback from an external cavity containing a quarter-wavelength plate, are

$$
\begin{aligned}
\dot{E}_{x}= & -\left(k+\gamma_{a}\right) E_{x}-i\left(\alpha k+\gamma_{p}\right) E_{x} \\
& +k(1+i \alpha)\left(N E_{x}+i n E_{y}\right)-\gamma E_{y}(t-\tau) e^{i \omega_{o} \tau}, \\
\dot{E}_{y}= & -\left(k-\gamma_{a}\right) E_{y}-i\left(\alpha k-\gamma_{p}\right) E_{y} \\
& +k(1+i \alpha)\left(N E_{y}-i n E_{x}\right)+\gamma E_{x}(t-\tau) e^{i \omega_{o} \tau}, \\
\dot{N}= & -\gamma_{N}\left[N\left(1+\left|E_{x}\right|^{2}+\left|E_{y}\right|^{2}\right)-J+i n\left(E_{x} E_{y}^{*}-E_{y} E_{x}^{*}\right)\right], \\
\dot{n}=- & \gamma_{s} n-\gamma_{N}\left[n\left(\left|E_{x}\right|^{2}+\left|E_{y}\right|^{2}\right)+i N\left(E_{y} E_{x}^{*}-E_{x} E_{y}^{*}\right)\right] .
\end{aligned}
$$

Here, $E_{x}$ and $E_{y}$ are $x$ - and $y$-polarized components of the slowly varying amplitude of the electric field (written in the $x-y$ basis of the VCSEL). The field amplitude is coupled to two population inversion variables: $N$ is the total population difference between the conduction and valence bands, and $n$ is the population difference between the carrier densities with positive and negative spin values. The parameters in these equations are as follows: $\alpha$ is the linewidth enhancement factor, $\omega_{o}$ is the solitary laser frequency in the absence of linear phase anisotropy, $\gamma_{a}$ is the anisotropic field loss rate (positive $\gamma_{a}$ gives the $y$-polarized component a lower threshold), $\gamma_{p}$ leads to a frequency difference of $2 \gamma_{p}$ between the $x$ - and $y$-polarized solutions in the absence of external feedback. $J$ is the normalized injection current (which takes the value 1 at the lasing threshold). The model includes several decay rates. $k$ is the decay rate of the electric field in the cavity, $\gamma_{N}$ is the decay rate of the total carrier number, and $\gamma_{s}$ is the decay rate that accounts for the mixing of the populations with different spins (usually due to the spin-flip relaxation processes). The external cavity is represented by two parameters: $\gamma$, which is the feedback strength, and $\tau$ $=2 \mathrm{~L} / \mathrm{c}$, which is the round-trip time in the extended cavity of length $L$.

We solved Eqs. (1)-(4) with weak stochastic noise perturbations added to each of the variables ${ }^{6}$ and an integration step of $0.5 \mathrm{ps}$. The parameters were chosen in our simulation to match those of the experimental situation of Ref. 1: $\alpha$ $=3, \omega_{o} \tau=6 \mathrm{rad}, \gamma_{a}=0.5 \mathrm{~ns}^{-1}, \gamma_{p}=40 \mathrm{~ns}^{-1}, k=300 \mathrm{~ns}^{-1}$, $\gamma_{N}=1 \mathrm{~ns}^{-1}$, and $\gamma_{s}=50 \mathrm{~ns}^{-1}$. For these parameters the split- 


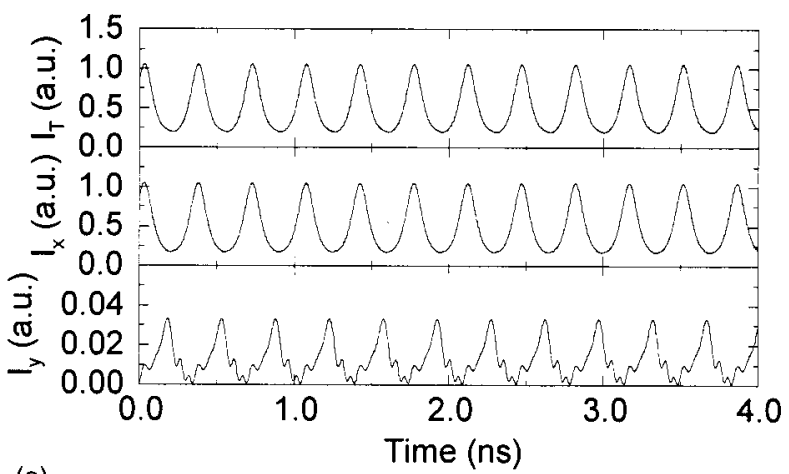

(a)

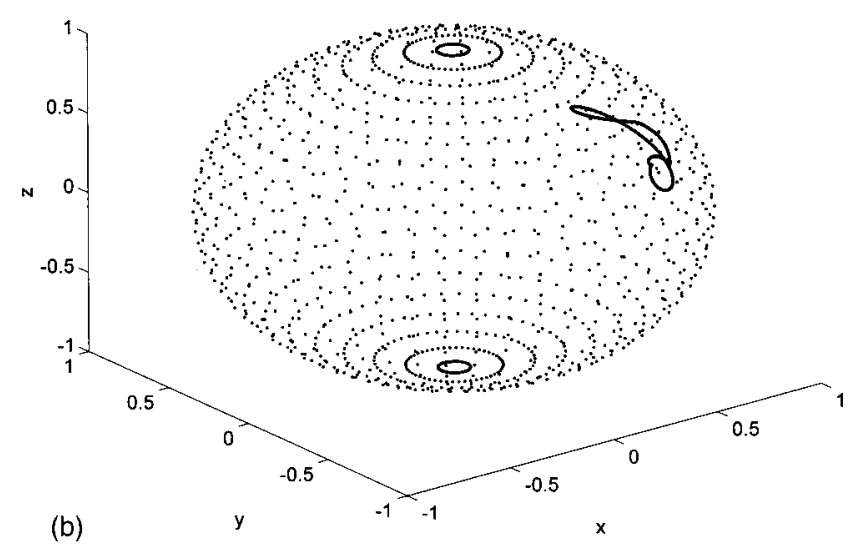

FIG. 1. Total intensity and intensities of $x$ - and $y$-polarized components of the solution, when $\gamma=10 \mathrm{GHz}$ and $\tau=0.2 \mathrm{~ns}$. Representation on the Poincare sphere (the dots indicate contours of constant latitude).

ting of the solitary VCSEL's linearly polarized eigenstates is about $12 \mathrm{GHz}$, the $y$-polarized solution has a lower threshold, and the laser switches from the $y$-polarized to the $x$-polarized state at about $J=1.2$. The solutions were calculated for $J$ $=1.5$ for which, in the absence of feedback, the $x$-polarized solution is the only stable solution. ${ }^{6}$

In the presence of optical feedback we find distinct dynamical regimes, depending on the feedback level. To characterize the polarization state of the light we use the Poincaré-sphere plot, where for a given pair of field amplitude components $E_{x}(t) E_{y}(t)$, we assign a point on the Poincaré-sphere whose coordinates are

$x=\frac{\left|E_{x}\right|^{2}-\left|E_{y}\right|^{2}}{\left|E_{x}\right|^{2}+\left|E_{y}\right|^{2}}, \quad y=\frac{2 \operatorname{Re}\left[E_{x} E_{y}^{*}\right]}{\left|E_{x}\right|^{2}+\left|E_{y}\right|^{2}}, \quad z=\frac{2 \operatorname{Im}\left[E_{x} E_{y}^{*}\right]}{\left|E_{x}\right|^{2}+\left|E_{y}\right|^{2}}$.

The points in the Poincare sphere of unit radius are in one-to-one correspondence with the different polarization states of the laser beam (the south pole represents leftcircular polarization, the north pole, right-circular polarization, the positive $x$ axis represents $x$ polarization while the negative $x$ axis represents $y$ polarization). The Poincarésphere representation does not conceal the trivial time dependence of a frequency difference between two spectral components, since a constant-intensity solution appears as a dot on the sphere (with longitude and latitude determined by the state of polarization), while the superposition of two spectral components with different polarization states appears as a circle on the Poincare sphere (centered on an axis that connects the two polarization states).

Figs. 1-4 show the intensity wave forms and their rep-

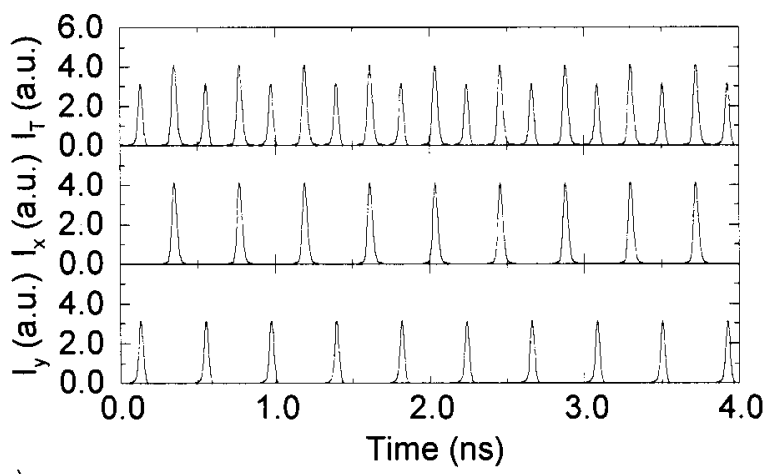

(a)

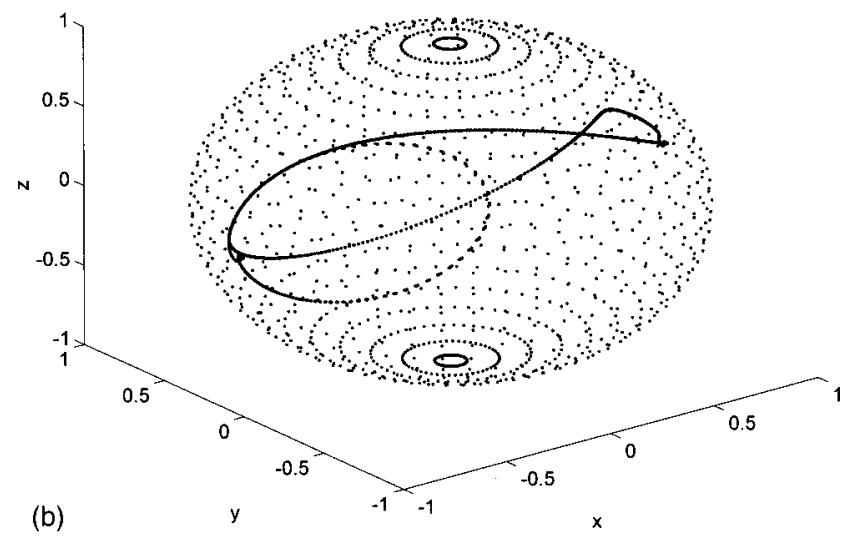

FIG. 2. Same as Fig. 1 but for $\gamma=50 \mathrm{GHz}, \tau=0.2 \mathrm{~ns}$.

resentation on the Poincare sphere. ${ }^{6}$ For very low-feedback levels the $x$ - and $y$-polarized intensities are constant, with $I_{y}$ much lower than $I_{x}$, indicating that weak feedback shifts the steady state to a slightly elliptically polarized state. While the linearly polarized steady-state solutions of Eqs. (1)-(4) with $\gamma=0$ have different optical frequencies (because of the birefringence), optical feedback and the nonlinear dispersive properties of the medium can force the total emission to be a "hybrid" of the two modes, that is steady-state (constant intensity) emission of an elliptically polarized mode.

Increasing the feedback above a critical value results in periodic modulation that begins as an infinitesimal modulation of the intensity and ellipticity (Fig. 1). The period of the modulation is nearly equal to the period of the relaxation oscillations of the solitary single-mode laser $\tau_{R}$ $=2 \pi / \sqrt{2 k \gamma_{N}(J-1)} \approx 0.36 \mathrm{~ns}^{5}$

For moderate feedback levels chaotic fluctuations (that are relatively anticorrelated) of the two nonlinear $x$ - and $y$-polarized modes occur. For higher feedback short pulses separated by one cavity round-trip time appear in the total intensity, which alternate in the relative intensity of the $x$ and $y$-polarized modes (Fig. 2).

Further increase of the feedback intensity leads to a new sinusoidal modulation (Fig. 3). The amplitudes of the modulations of the $x$ - and $y$-polarized intensities are nearly the same, and the period is slightly larger than twice the external cavity round-trip time. This appears to represent (predominantly) the beating between two orthogonal elliptically polarized modes of different optical frequencies. For a larger delay time the modulation becomes approximately a square wave (Fig. 4). These computed solutions of modulated be- 


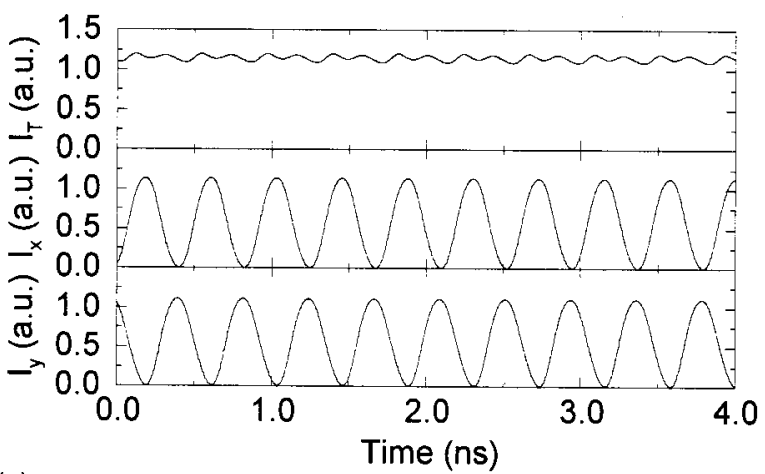

(a)

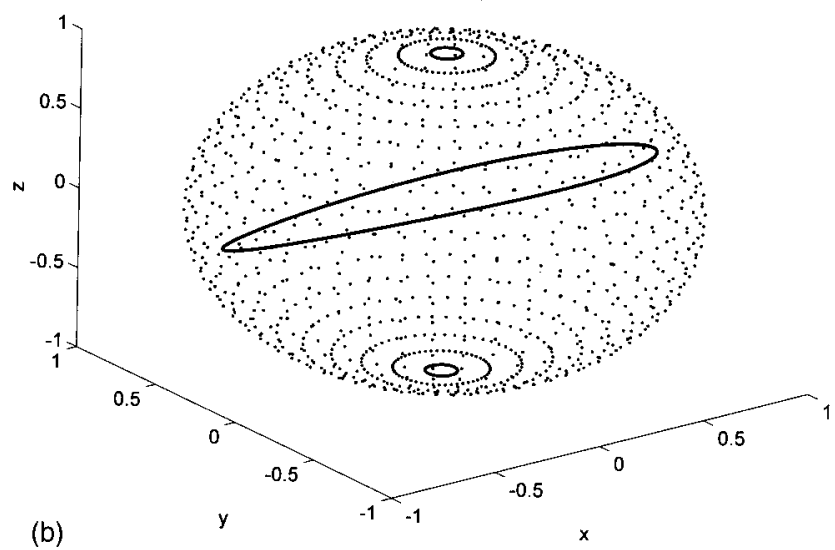

FIG. 3. Same as Fig. 1 but for $\gamma=100 \mathrm{GHz}, \tau=0.2 \mathrm{~ns}$.

havior agree with those reported in the experiments (Ref. 1, Fig. 3).

Similar results were obtained for different external cavity lengths. However, the larger the external cavity length, the narrower the feedback interval in which the periodic modulation of the $x$ - and $y$-polarized intensities occurs (we have studied external cavity delay times less than $1 \mathrm{~ns}$ since the small integration step needed for the fast dynamical behavior would require excessively long computations for large values of $\tau$ ).

The sinusoidal modulation is rather insensitive to variations of the feedback phase $\omega_{o} \tau$ (i.e., variations of the position of the external mirror on the order of a wavelength), but the square modulation changes its shape with variations of $\omega_{o} \tau$ (for certain values of $\omega_{0} \tau$ the dynamics is chaotic).

The dynamics depend strongly on the value of the spin relaxation rate $\gamma_{s}$. For the parameters used in Figs. 3 and 4, but lower values of $\gamma_{s}\left(\gamma_{s}<10 \sim 20\right)$, only chaotic competition between the $x$ - and $y$-polarized modes occurs. In the limiting case of very fast spin relaxation $\left[\gamma_{s} \rightarrow \infty\right.$, which corresponds to setting $n=0$ in Eqs. (1)-(4)], and for feedback levels higher than those causing chaotic competition, the only wave forms found were sinusoidal modulation of the intensities of both linearly polarized components (in some cases, an elliptically polarized steady state is reached after a long sinusoidal transient). This suggests that a model in which the two modal amplitudes $E_{x}, E_{y}$ are coupled to a single-carrier population $N$ fails to explain all the experimental features of the dynamics. Intermediate values of $\gamma_{s}$ in the model of San Miguel et al. (or some equivalent dynamical lethargy and coupling) are needed in order to reproduce the square-wave behavior.

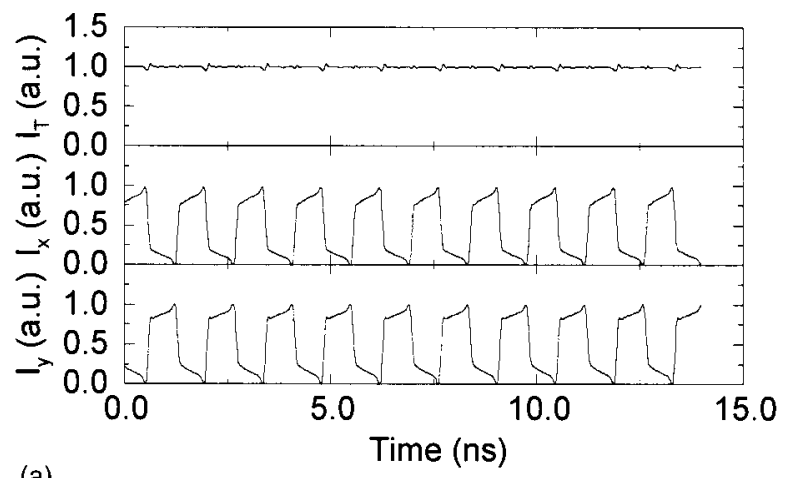

(a)

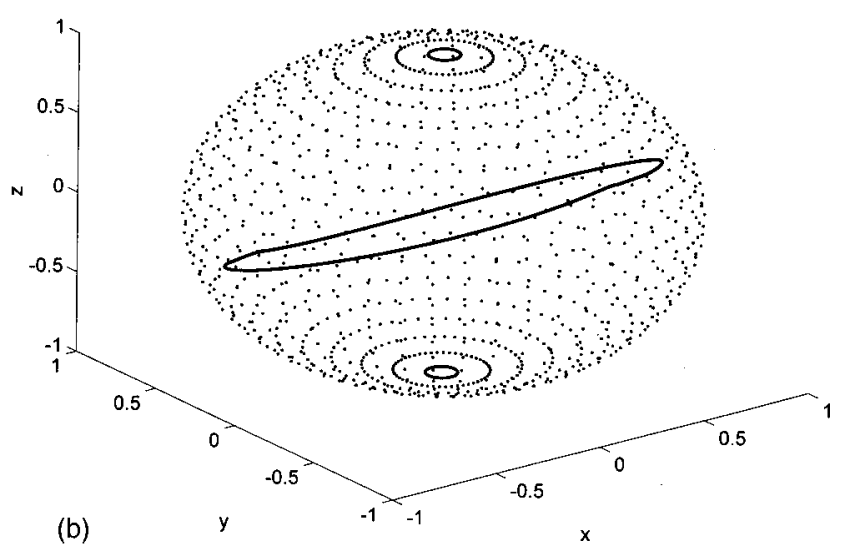

FIG. 4. Same as Fig. 1 but for $\gamma=85 \mathrm{GHz}, \tau=0.7 \mathrm{~ns}$.

In summary, VCSELs have shown clear evidence of birefringence and gain anisotropy, but simple models that only take into account these characteristics of the semiconductor medium fail to explain all the experimental findings. We show that subtleties of the laser physics can be explained by including additional physically realistic and polarizationsensitive variables and their relaxation rates. Using the fourlevel model of San Miguel, Feng, and Moloney of a quantum-well VCSEL to study the response of a VCSEL to optical feedback from an external cavity containing a quarter-wavelength plate, we have shown that for strong feedback levels the computed results are in good agreement with the experimental findings of Ref. 1. Since in Ref. 1 the experiments were performed under a high, fixed feedback level, it would be interesting to have experimental results of the VCSEL dynamics with weak and moderate feedback to compare with our predictions, in order to test the broader validity of the model.

This work was supported by the Comisión Sectorial de Investigación Científica (CSIC), and the Proyecto de Desarrollo de Ciencias Básicas (PEDECIBA).

${ }^{1}$ S. Jiang, Z. Pan, M. Dagenais, R. A. Morgan, and K. Kojima, Appl. Phys. Lett. 63, 3545 (1993)

${ }^{2}$ Z. G. Pan, S. Jiang, M. Dagenais, R. A. Morgan, K. Hojima, M. T. Asom, and R. E. Leibenguth, Appl. Phys. Lett. 63, 2999 (1993).

${ }^{3}$ K. D. Choquette, K. L. Lear, R. E. Leibenguth, and M. T. Asom, Appl. Phys. Lett. 64, 2767 (1994)

${ }^{4}$ F. Robert, P. Besnard, M.-L. Chares, and G. M. Stephan, Opt. Quantum Electron. 27, 805 (1995).

${ }^{5}$ M. San Miguel, Q. Feng, and J. V. Moloney, Phys. Rev. A 52, 1729 (1995).

${ }^{6}$ J. Martin-Regalado, F. Prati, M. San Miguel, and N. B. Abraham, IEEE J. Quantum Electron. 33, 765 (1997). 